\title{
Percutaneous antegrade nephroscopic holmium laser pyelotomy: Novel endourologic technique for removal of extruded ureteral stent
}

\author{
Jonathon Rhee, MD; Stephen S. Steele, MD, FRCSC; Darren Beiko, MD, FRCSC
}

Department of Urology, Queen's University, Kingston General Hospital, Kingston, ON

Cite as: Can Urol Assoc J 2013;7(11-12):e830-2. http://dx.doi.org/10.5489/cuaj.1258 Published online December 5, 2013.

\section{Abstract}

Ureteral stent malposition outside of the urinary tract is a very uncommon complication of retrograde or antegrade ureteral stent insertion. There are few reports of open, laparoscopic or endourologic approaches to remove malpositioned stents. We present a novel technique for the removal of an extruded retroperitoneal ureteral stent using percutaneous antegrade nephroscopic holmium laser pyelotomy. This previously undescribed procedure represents a new soft tissue application of the holmium laser.

$\mathrm{R}$ etrograde or antegrade ureteral stent placement is a common practice to relieve upper urinary tract obstruction, pain, or infection. ${ }^{1}$ Minor complications of ureteral stents include pain, voiding symptoms, hematuria and infection, and major complications of stents include encrustation, fragmentation, migration, malposition, knot formation, forgotten stents, extrusion and death. ${ }^{1-3}$ Ureteral stent migration is rare and involves movement within the urinary system. Stent extrusion is extremely rare and involves movement outside of the urinary tract, sometimes into critical organs such as the inferior vena cava ${ }^{4,5}$ or duodenum. ${ }^{6}$ Ureteral stent malposition is uncommon because of imaging techniques that are widely employed during stent placement. Ureteral stent malposition contained within the urinary tract lumen is often identified intraoperatively. However, malposition and extrusion of a ureteral stent outside of the urinary tract is extremely rare and may or may not be identified at time of stent insertion. We report a rare case of an antegrade malpositioned and extruded ureteral stent outside of the renal pelvis and its unique successful management via percutaneous antegrade nephroscopic holmium:yttrium aluminium garnet (YAG) laser pyelotomy - a procedure that has not been reported in the literature to date.

\section{Case report}

A 78-year-old female presented with acute renal failure and bilateral hydronephrosis secondary to an obstructing, locally advanced and metastatic colorectal carcinoma. Serum creatinine was $922 \mu \mathrm{mol} / \mathrm{L}$. Bilateral nephrostomy tubes and antegrade $8.5 \mathrm{Fr}$ double J ureteral stents were placed by the interventional radiology service at our institution under ultrasonographic and fluoroscopic guidance. Within 3 days, the patient's creatinine returned to baseline $(54 \mu \mathrm{mol} / \mathrm{L})$ and both nephrostomy tubes were removed 5 days later. The patient was discharged in stable condition with indwelling bilateral ureteral stents. At initial follow-up 6 weeks after stent insertion, the patient reported mild intermittent gross hematuria, but no nausea or stent colic. Serum creatinine remained normal.

Elective bilateral ureteral stent change was attempted under general anaesthesia 3 months later. Left-sided stent change was uneventful, despite a partially encrusted stent. The right-sided stent was not changed due to significant resistance that was met while attempting to remove it. The procedure was abandoned and a kidneys-ureters-bladder $(K \cup B) x$-ray showed abnormal coiling of the proximal end of the stent (Fig. 1). Abdominopelvic computed tomography (CT) scan revealed malposition of the proximal right ureteral stent coil in the retroperitoneum, posterior to the renal pelvis but within the perirenal adipose tissue (Fig. 2). This explained the resistance that was met when prior attempt was made to remove the right ureteral stent. No other abnormalities were noted.

Laparoscopic, open, ureteroscopic and percutaneous approaches were considered and discussed with the patient. Informed consent was obtained for percutaneous antegrade nephroscopic stent retrieval and replacement, including laser pyelotomy if necessary. The procedure was performed under general anesthesia in the prone position with $1 \mathrm{~g}$ cephazolin administered intravenously preoperatively. Prone cystoscopy 


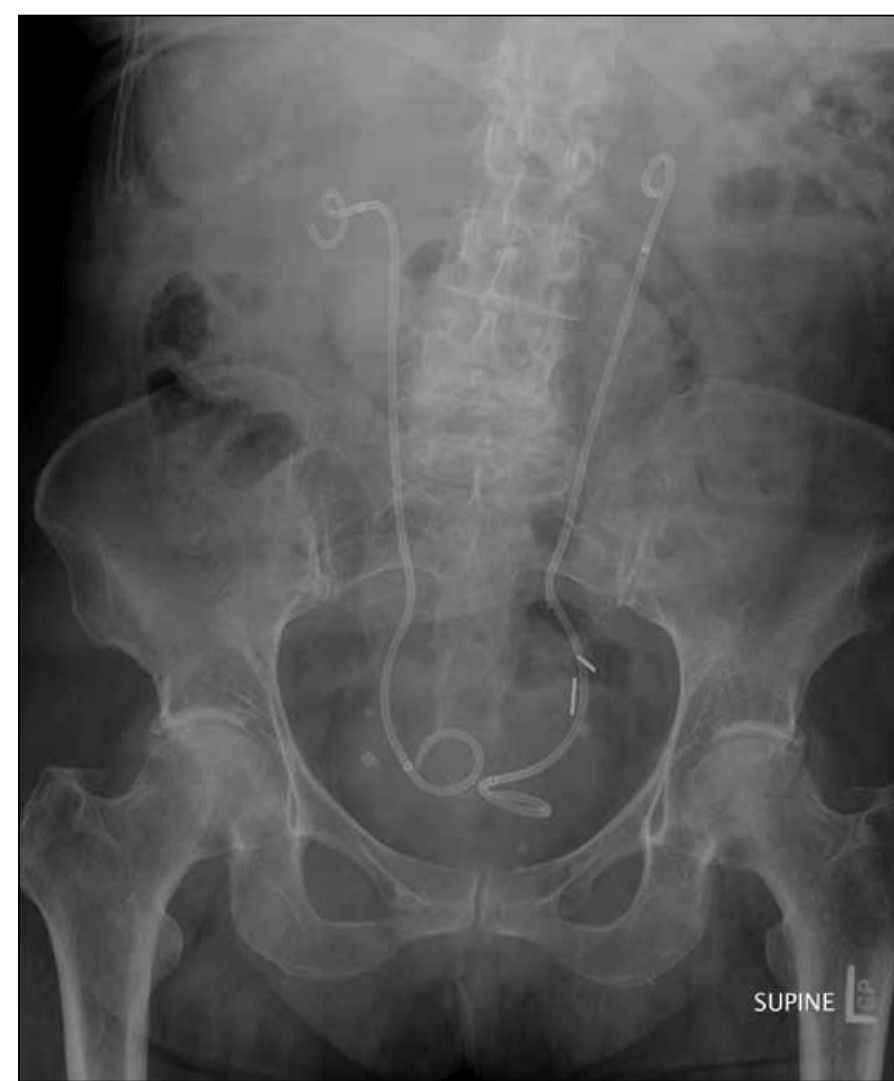

Fig. 1. Kidneys-ureters-bladder (KUB) $x$-ray showing abnormal proximal coil of the right ureteral stent.

and right retrograde ureteral access were achieved. Right retrograde pyelogram (RGP) confirmed the presence of the extruded segment of stent posterior to the renal pelvis (Fig. $3)$. Interpolar percutaneous access was achieved and the tract was dilated by balloon technique and a 24-Fr rigid nephroscope was used through a 30-Fr sheath to visualize the renal pelvis and the site of the extruded stent - on the posterior aspect of the renal pelvis. The proximal end of the stent where it exited the renal pelvis was fixed and did not release with gentle pulling using alligator forceps. Therefore, the shaft of the stent was grasped at the ureteropelvic junction (UPJ) and the entire distal end of the stent was pulled up through the UPJ and nephrostomy sheath and secured, providing tension at the point of extrusion in the renal pelvis. A holmium:YAG $910 \mathrm{~nm}$ laser fibre was introduced through the nephroscope, and settings of 1.5 Joules and 10 Hertz (15 watts) were used to perform a circumferential pyelotomy incision around the base of the site of stent extrusion. This approach was successful at incising the tissue and the stent was liberated in its entirety and safely removed out of the body through the nephrostomy sheath. Surprisingly, antegrade nephrostogram did not show any extravasation. A new ureteral stent was placed in an antegrade fashion over

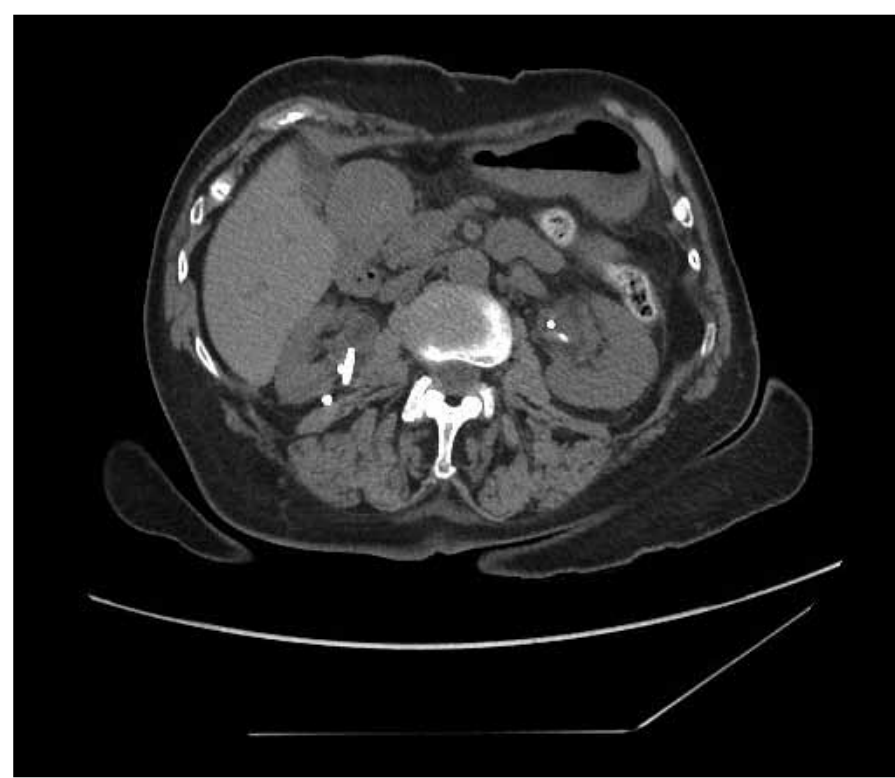

Fig. 2. A computed tomography scan showing malposition of the proximal end of the stent posterior to the renal pelvis.

a wire and placement was confirmed with fluoroscopy. In the absence of extravasation and bleeding, the patient was left without a nephrostomy tube.

There were no postoperative complications and the patient was discharged in stable condition with oral antibiotics and oral narcotic analgesics. After 2 years of followup, the patient is receiving palliative care for her metastatic colorectal carcinoma, and has undergone 2 ureteral stent changes. Her renal function remains stable and there have been no complications related to her stents, which were converted over to long-term indwelling Inlay Optima (Bard Medical Division, Covington, GA) stents requiring only annual exchange.

\section{Discussion}

Ureteral stent malposition or extrusion is an uncommon complication. Various techniques (including open,, 78 laparoscopic ${ }^{9}$ and endourologic ${ }^{10}$ approaches) have been described, usually in isolated case reports. In this case, we opted for a percutaneous approach because it was more definitive than a retrograde ureterorenoscopic approach and it spared the patient the morbidity of an open procedure.

We hypothesize that the most plausible explanation for the malpositioned antegrade stent in our case was a "through-and-through" collecting system access - initial calyceal puncture, followed by transient passage of needle or guidewire outside the collecting system and immediately back in through the posteroinferior aspect of the renal pelvis. Unfortunately, this was not initially identified at time of stent placement. But thankfully, the malpositioned stent 


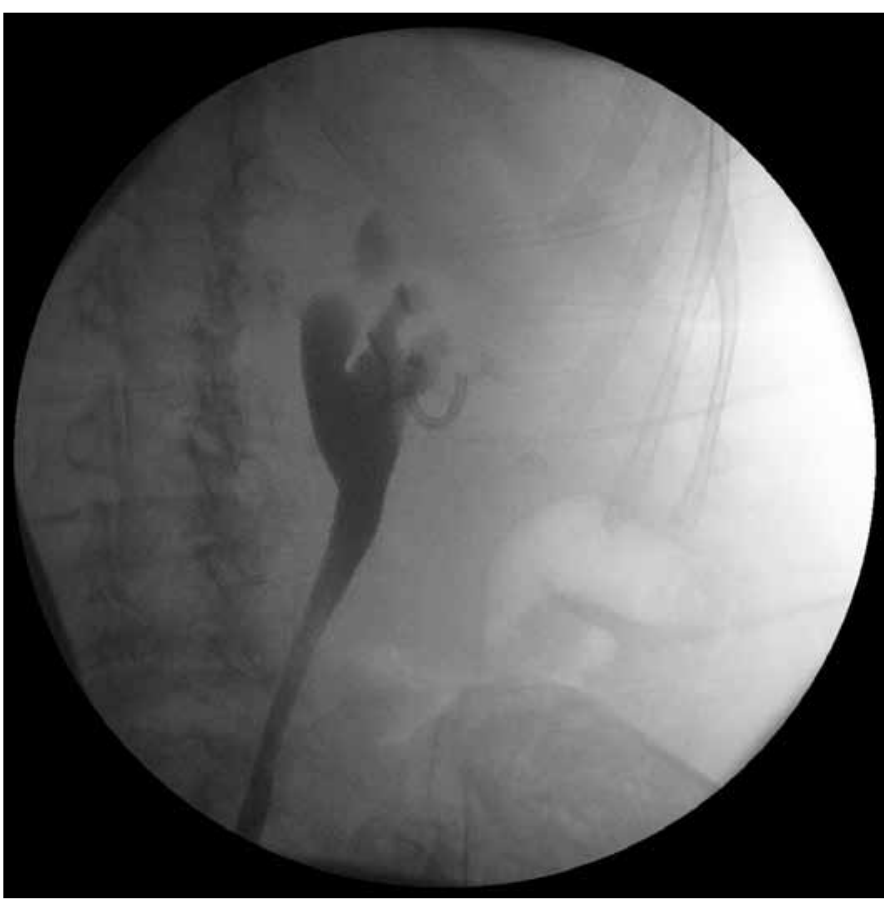

Fig. 3. Intraoperative fluoroscopic retrograde pyelogram image showing tip of stent emanating from posteroinferior aspect of renal pelvis.

extruded posterior to the patient's renal pelvis, permitting a minimally invasive endourologic approach. The posterior location was away from the renal vessels, thus allowing a safe percutaneous approach, including laser pyelotomy. Due to the close proximity of the main renal vessels and segmental branches, we do not recommend laser pyelotomy for any anteriorly-oriented extruded stents unless a preoperative CT scan clearly shows the site of extrusion to be well away from the vessels.

Our minimally invasive novel technique was successful, as the patient had an uneventful recovery from her procedure and continues to do well in postoperative follow-up. This novel use of the Holmium:YAG to perform a percutaneous antegrade endoscopic laser pyelotomy to retrieve a malpositioned stent outside the renal pelvis has not been reported before. The application of the Holmium:YAG laser within the realm of urology, given its precision in dealing with soft tissue, has expanded beyond lithotripsy; this procedure is yet one additional application.

\section{Conclusion}

In order to spare patients the morbidity of an open or laparoscopic procedure, percutaneous holmium laser pyelotomy should be considered for the retrieval of malpositioned or extruded stents. This is one more technique to add to the (endo)urologist's armamentarium.

Competing interests: Dr. Rhee, Dr. Steele and Dr. Beiko all declare no competing financial or personal interests.

This paper has been peer-reviewed.

\section{References}

1. Duvdevani $M$, Chew BH, Denstedt JD. Minimizing symptoms in patients with ureteric stents. Curr Opin Urol 2006; 16:77-82. http://dx.doi.org/10.1097/01.mou.0000193375.29942.0f

2. Singh $V$, Srinivastava $A$, Kapoor $R$, et al. Can the complicated forgotten indwelling ureteric stents be lethal? Int Urol Nephrol 2005;37:541-6. http://dx.doi.org/10.1007/s1 1255-004-4704-6

3. Karagüzel E, Kutlu 0, Kazaz 10, et al. Knotted ureteral stent: a rare complication of ureteral stent usage. Urol Res 2012;40:793-5. http://dx.doi.org/10.1007/s00240-012-0496-9

4. Falahatkar S, Hemmati H, Gholamiani Moghaddam K. Intracaval migration: an uncommon complication of ureteral Double-J stent placement. J Endourol 2012;26:119-21. http://dx.doi.org/10.1089/ end.2011.0469

5. loannou CV, Velegrakis J, Kostas T, et al. Caval migration of a ureteral J-stent after simultaneous ureter and liliac vein perforation during its placement for obstructive pyelonephritis. Int Angiol 2009;28:421-4.

6. Wall I, Baradarian $R$, Tangorra $M$, et al. Spontaneous perforation of the duodenum by a migrated ureteral stent. Gastrointest Endosc 2008;68:1236-8. http://dx.doi.org/10.1016/i.gie.2008.02.083

7. Shivde SR, Joshi P, Jamkhandikar R. Extrusion of double I stent: a rare complication. Urology 2008;71:814-5. hittp://dx.doi.org/10.1016/i.urology.2008.02.017

8. loannis C, loannis Z, Evangelos I, et al. Retrovesical migration of malpositioned double-j ureteral stent. Int Urol Nephrol 2003;35:325-6. http://dx.doi.org/10.1023/B:UROL.0000022913.96740.e8

9. Abraham G, Das K, George D. Retroperitoneal migration of a Double-J stent: an unusual occurrence. J Endourol 2011;25:297-9. http://dx.doi.org/10.1089/end.2010.0281

10. Cormio L, Piccinni R, Cafarelli A, et al. Asymptomatic spontaneous migration of double pigtail ureteral stent outside the ureter. Int Urol Nephrol 2007;39:75-7. http://dx.doi.org/10.1007/s11255-005-4757-1

Correspondence: Dr. Darren Beiko, Associate Professor, Department of Urology, Queen's University, Kingston General Hospital, 76 Stuart St., Kingston, ON K7L 2V7; fax: 613-545-1970; beikod@kgh.kari.net 